appear to contain cloning artifacts.

13. The $5^{\prime}$ end of the human cDNA was obtained by two rounds of PCR amplification of the CLN2 can didate from a human cortex cDNA library (Stratagene) by means of two different gene-specific primers and a single vector-specific primer. In the first round of PCR the T3 promoter primer was used with either gene-specific primer NR1 (5'-GTGATCACAGAATGGCACTT) or NR2 (5'-AACATGGGTTTCCGTAGGTC). In the second round of PCR, with the products from the first amplification, the T3 promoter primer and NR4 (5'-CTTCCTCAGGGTCCGCACGG) were used.

14. GenBank accession number AF017456.

15. Three lines of evidence give corroborative results for an unequivocal localization. (i) There is a nearly perfect match between nt 34 to 104 of the CLN2 cDNA candidate and GenBank accession number B04497, which represents a PCR-amplified fragment of a flow-sorted chromosome 11-specific cosmid clone. (The 317-nt B04497 also contains sequence of flanking introns.) (ii) There is a perfect 505-nt match between the $3^{\prime}$ end of the CLN2 cDNA (nt 2979 to 3483) and the $5^{\prime}$ end (nt 1 to 505) of GenBank accession number U25816. U25816 consists of 2605 nt that encompass the human TATAbinding protein associated factor $\| 30\left(\mathrm{TAF}_{\|} 30\right)$ gene. The $\mathrm{TAF}_{\|} 30$ transcription start site is at U25816 nt 1060, and most of the promoter elements are downstream of U25816 nt 860 and thus do not overlap with the $3^{\prime}$ end of the large CLN2 candidate transcript. Thus, the CLN2 candidate gene and the $\mathrm{TAF}_{\|} 30$ gene are physically adjacent. The $\mathrm{TAF}_{\|} 30$ gene was mapped to chromosome 11p15.2-p15.5 by means of in situ hybridization [E. Scheer, M. G. Mattei, X. Jacq, P: Chambon, L. Tora, Genomics 29 269 (1995)]. (iii) Three sequences (accession numbers $X 72877, X 72878$, and $X 72880$ ) representing a cosmid clone have strong matches $\left(P<10^{-31}\right)$ to $n$ 2817 to 3264 of the CLN2 candidate cDNA. The cosmid clone maps to chromosome 11p15. Taken together, these results indicate that the CLN2 candidate is localized to chromosome 11p15.

16. D. E. Sleat et al., unpublished data.

17. CLN2 was analyzed in patient DNA extracted from cell lines by means of overlapping M13 forward-and reverse-tailed primer pairs. Each pair amplified an exon and flanking intronic sequences, and the resulting products were sequenced with dye-labeled $-21 \mathrm{M} 13$ primer. For patients, the sequence of frag ments that mismatched with the consensus sequence was then confirmed by sequencing with the M13 reverse primer. Each fragment containing a mu tation in both patients and relatives was then independently reamplified and sequenced on both strands to confirm that the observed heterogeneities were not artifacts of PCR amplification. Primer pairs that detected mutations in patient DNA were SF3 (5'-TGTAAAACGACGGCCAGTCAGACCTTCCAG TAGGGACC)/SR3 (5'-CAGGAAACAGCTATGAC CCTGTATCCCACACAAGAGAT) and SFOA (5' TGTAAAACGACGGCCAGTTAGATGCCATTGGG GACTGG)/SROA (5'-CAGGAAACAGCTATGAC CGTCATGGAAATACTGCTCCA). PCR from 1- $\mu \mathrm{g}$ samples of patient DNA with Vent DNA polymerase (New England Biolabs, Beverly, MA) was done under the following cycle conditions: $94^{\circ} \mathrm{C}$ for $3 \mathrm{~min}$ followed by 10 cycles of $94^{\circ} \mathrm{C}$ for $1 \mathrm{~min}, 50^{\circ} \mathrm{C}$ for $1 \mathrm{~min}$ and $72^{\circ} \mathrm{C}$ for $1 \mathrm{~min}$, followed by 30 cycles of $94^{\circ} \mathrm{C}$ for $1 \mathrm{~min}, 65^{\circ} \mathrm{C}$ for $1 \mathrm{~min}$, and $72^{\circ} \mathrm{C}$ for $1 \mathrm{~min}$, with a final incubation for $10 \mathrm{~min}$ at $72^{\circ} \mathrm{C}$. Products were purified by means of Qiaquick spin columns (Quiagen Chatsworth, CA) and cycle-sequenced with AmpliTaq DNA polymerase (Roche Molecular Systems, Alameda, $\mathrm{CA}$ ) and $\mathrm{AB}$ I Prism dye-labeled primers (Perkin Elmer, Foster City, CA) on an ABI 373 automated sequencer.

18. A BLAST search of the SwissProt database with the conceptually translated CLN2 candidate gave a highly significant match with PsCP: $P=1.9 \times$ $10^{-11}$; the Dayhoff comparison score is $>8$ SDs above the mean (ALIGN program, relative to 200 comparisons of scrambled sequences); and pairwise comparison with GCG Bestfit yields identity and similarity scores of 25 and $46 \%$, respectively.

PsCP is related (52\% identical, $66 \%$ similar) to $\mathrm{XaCP} . \mathrm{XaCP}$ is not detected in a BLAST search with the CLN2 candidate, but in pairwise comparisons the Dayhoff comparison score is $>2.7$ SDs above the mean, and the identity and similarity scores are 24 and $48 \%$, respectively.

19. K. Oda, T. Takahashi, Y. Tokuda, Y. Shibano, S. Takahashi, J. Biol. Chem. 269, 26518 (1994).

20. K. Oda et al., J. Biochem. 120, 564 (1996).

21. D. N. Palmer, I. M. Fearnley, S. M. Medd, Am. J. Med. Genet. 42, 561 (1992).

\title{
Mutation of the Stargardt Disease Gene (ABCR) in Age-Related Macular Degeneration
}

\author{
Rando Allikmets, ${ }^{*}$ Noah F. Shroyer, ${ }^{*}$ Nanda Singh, ${ }^{*}$ \\ Johanna M. Seddon, Richard Alan Lewis, Paul S. Bernstein, \\ Andy Peiffer, Norman A. Zabriskie, Yixin Li, Amy Hutchinson, \\ Michael Dean, $\dagger$ James R. Lupski, Mark Leppert
}

Age-related macular degeneration (AMD) is the leading cause of severe central visual impairment among the elderly and is associated both with environmental factors such as smoking and with genetic factors. Here, 167 unrelated AMD patients were screened for alterations in $A B C R$, a gene that encodes a retinal rod photoreceptor protein and is defective in Stargardt disease, a common hereditary form of macular dystrophy. Thirteen different AMD-associated alterations, both deletions and amino acid substitutions, were found in one allele of $A B C R$ in 26 patients (16\%). Identification of $A B C R$ alterations will permit presymptomatic testing of high-risk individuals and may lead to earlier diagnosis of AMD and to new strategies for prevention and therapy.

Age-related macular degeneration (AMD) is the most common cause of acquired visual impairment in the elderly and is estimated to affect at least 11 million individuals in the United States (1-3). U.S. studies show that mild forms of AMD occur in nearly 30\% of those 75 years and older, and advanced forms occur in about $7 \%$ of people in this age group (2). Clinically, AMD is divided into two

R. Allikmets, Intramural Research Support Program, SAIC-Frederick, NCl-Frederick Cancer Research and Development Center, Frederick, MD 21702, USA.

N. F. Shroyer and Y. Li, Department of Molecular and Human Genetics, Baylor College of Medicine, Houston, TX 77030, USA

N. Singh, A. Peiffer, M. Leppert, Department of Human Genetics, Eccles Institute of Human Genetics, University of Utah, Salt Lake City, UT 84112, USA.

J. M. Seddon, Department of Ophthalmology, Massachusetts Eye and Ear Infirmary, Harvard Medical School, Boston, MA 02114, USA.

R. A. Lewis, Departments of Molecular and Human Genetics, Ophthalmology, Pediatrics, and Medicine, Baylor College of Medicine, Houston, TX 77030, USA

P. S. Bernstein and N. A. Zabriskie, Department of Ophthalmology, Moran Eye Center, University of Utah, Salt Lake City, UT 84132, USA.

A. Hutchinson and M. Dean, Laboratory of Genomic Diversity, $\mathrm{NCl}$-Frederick Cancer Research and Development Center, Building 560, Room 21-18, Frederick, MD 21702-1201, USA

J. R. Lupski, Departments of Molecular and Human Genetics and Pediatrics, Baylor College of Medicine, Houston, TX 77030, USA.

These authors contributed equally to this work. †To whom correspondence should be addressed. E-mail: dean@fcrtv1.ncifcrf.gov subtypes: $80 \%$ of patients have "dry" AMD, the hallmarks of which include one or more of the following: the presence of cellular debris (drusen) in or under the retinal pigment epithelium (RPE), irregularities in the pigmentation of the RPE, or geographic atrophy; $20 \%$ of patients have exudative or "wet" AMD, characterized by serous detatchment of the RPE or choroidal neovascularization or both. Severe vision loss is associated most often with geographic atrophy or exudative disease $(4,5)$. There are no reliable therapies for dry AMD, and only about $5 \%$ of patients with the wet subtype are candidates for laser photocoagulation therapy (4).

AMD is a multifactorial disorder that is associated with environmental risk factors such as cigarette smoking, diet, and cholesterol level $(3,6)$. Genetic factors also contribute to $\operatorname{AMD}(7,8)$, although genetic studies have been confounded by the late onset and complex etiology of the disease. One approach to identifying the genes responsible for multifactorial disorders is to study inherited diseases with similar phenotypes. Several hereditary retinal dystrophies have phenotypic similarities to AMD. Sorsby fundus dystrophy, for example, resembles wet AMD and is caused by mutations in the tissue inhibitor of metalloproteinases-3 (TIMP3) gene (9). However, no 
mutations in TIMP3 or other retina-specific genes [for example, those for peripherin/ retinal degeneration slow (RDS) or rhodopsin] have been described in either wet or dry $\operatorname{AMD}(10,11)$.

We recently identified a photoreceptorspecific gene (ABCR, also known as STGD1) on human chromosome 1 p21 that is mutated in Stargardt disease (STGD1) (12). STGD1 is the most common form of hereditary, recessive macular dystrophy (estimated incidence, 1 in 10,000) and is characterized by juvenile-to-young adult onset, central visual impairment, progressive bilateral atrophy of the macular RPE and neuroepithelium, and the frequent appearance of orange-yellow flecks distributed around the macula and the midretinal periphery (13). The ABCR protein is a member of the adenosine triphosphate (ATP)-binding cassette $(A B C)$ transporter superfamily (12, 14), which includes active transporters of lipids, hydrophobic drugs, and peptides. ABCR corresponds to a previously identified rod outer segment protein called rim protein $(\mathrm{RmP})(15-17)$.

Age-related maculopathies have several phenotypic similarities with STGD1, including the accumulation of drusen in and under RPE and the progressive atrophy of the macular RPE (18). These changes result in loss of photoreceptor function and vision impairment. To test the hypothesis that $A B C R$ defects contribute to $A M D$, we screened for $A B C R$ sequence alterations in two independent collections of 96 and $71(\Sigma=167)$ unrelated AMD patients (19). Primers were designed to amplify each exon of $A B C R$ and its corresponding intron junctions.

Table 1. Prevalence of $A M D$-associated variants in $A B C R$ (23). NA, data not available.

\begin{tabular}{lccl}
\hline Mutation & $\begin{array}{c}\text { AMD } \\
(n=167)\end{array}$ & $\begin{array}{c}\text { STGD } \\
(n=98)\end{array}$ & $\begin{array}{c}\text { General } \\
\text { popula- } \\
\text { tion } \\
(n=220)\end{array}$ \\
\hline E471K & $2(1.2 \%)$ & NA & $0(0 \%)$ \\
R1129L & $1(0.6 \%)$ & $0(0 \%)^{*}$ & $0(0 \%)$ \\
T1428M & $1(0.6 \%)$ & $0(0 \%)$ & $0(0 \%)$ \\
R1517S & $1(0.6 \%)$ & $0(0 \%)$ & $0(0 \%)$ \\
I1562T & $2(1.2 \%)$ & $0(0 \%)$ & $0(0 \%)$ \\
G1578R & $1(0.6 \%)$ & $0(0 \%)$ & $0(0 \%)$ \\
$5196+1 G$ & $1(0.6 \%)$ & $0(0 \%)$ & $0(0 \%)$ \\
$\rightarrow$ A & $1(0.6 \%)$ & $4(4 \%)$ & $0(0 \%)$ \\
R1898H & $6(3.6 \%)$ & $8(8 \%)$ & $0(0 \%)$ \\
G1961E & $1(0.6 \%)$ & $0(0 \%)$ & $0(0 \%)$ \\
L1970F & $1(0.6 \%) \dagger$ & $1(1 \%) \dagger$ & $0(0 \%)$ \\
$6519 \Delta 11 b p$ & $7(4.2 \%)$ & $0(0 \%)$ & $1(0.45 \%)$ \\
D2177N & $1(0.6 \%)$ & $0(0 \%)$ & $0(0 \%)$ \\
$6568 \Delta \mathrm{C}$ & $26(16 \%)$ & $13(13 \%)$ & $1(0.45 \%)$ \\
Totals & 26 &
\end{tabular}

${ }^{*}$ A substitution to a different amino acid (R1129C) was detected in one STGD1 patient. †The two individuals with this variant are related (Fig. 2).
Single-strand conformation polymorphism (SSCP) and heteroduplex analysis (HA) were used to scan for variants; each alteration was characterized by direct sequencing (20). The exons found to be altered in AMD were also examined, by identical methodology, for variations in 98 unrelated STGD1 patients and in 220 racially matched population controls $(12,13,18$, 19).

All 51 exons (21) of ABCR were screened for variations in each AMD patient, and the results were grouped into AMD-associated alterations [those found in $A M D$ and not in controls or found significantly more frequently in AMD (Table 1)] and other alterations (Table 2). A total of 13 different AMD-associated alterations were detected in 26 AMD patients (16\%). Most were missense mutations in conserved amino acid positions, but two deletions representing frame shifts and one splice donor site change were found also (22) (Table 1). Although 33 of the 167 patients (20\%) had wet AMD, $A B C R$ alterations were found in only one such patient. Thus, this form of AMD may be associated with factors other than ABCR alterations. There was no obvious correlation between the type of $A B C R$ alterations and visual impairment, age of detection of retinal pathology, or other pathological parameters. Among the 26 individuals with alterations, 13 (50\%) had a first- or second-degree relative with AMD, which is significantly higher $(P<$ $0.005)$ than the fraction in the entire sample $(24 \%)$.

One alteration, $\mathrm{Asp}^{2177} \rightarrow \mathrm{Asn}^{2177}$ (D2177N) (23), was present in 7 of the 167
AMD patients and in only 1 of 220 controls, which is a significant difference by Fisher's exact test $(P=0.023)$. The associated retinal pathology ranged from fine macular cuticular drusen (age 62 years), to normal maculas but extensive extramacular and peripheral drusen (ages 72 and 74 years), to geographic atrophy involving the central third of the macula in each eye (ages 61 to 86 years). Six patients had a G1961E (23) alteration. The associated pathology ranged from a few tiny juxtafoveal drusen in one eye of one patient (age 74 years), to confluent drusen and drusenoid RPE detachments (age 78 years), to various forms of soft to calcific macular drusen and extensive geographic atrophy $(>1$ disc diameter) (ages 81 and 82 years). Other alterations were associated with various patterns of foveal, extrafoveal, and peripheral drusen, some with geographic atrophy of the RPE.

The AMD-associated alterations were scattered throughout the coding sequence of the $A B C R$ gene, although more were located toward the 3' end (Fig. 1). Three of the 13 alterations in AMD patients were also detected in STGD1 patients, which suggests that some mutations that cause recessive STGD1 may enhance susceptibility to AMD in the heterozygous state (24). Indeed, we identified two families in which the parents or grandparent of STGD1 probands have AMD (former pedigree, Fig. 2). Given the limited number of STGD1 families screened (98) [(12) and this study], it is unclear whether the remaining 10 alterations do not occur in STGD1 or whether they were simply not represented in our patient collection. In contrast to STGD1 mutations,

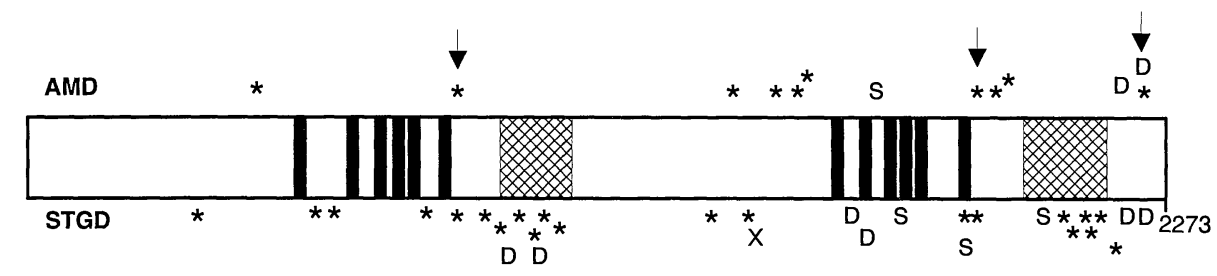

Fig. 1. Diagram of the $A B C R$ gene with STGD and $A M D$ alterations. Transmembrane domains predicted by hydropathy plot are shown as black bars, and the ATP-binding domains are shown as hatched bars. AMD-associated alterations are shown above the diagram, and those in STGD1 patients are shown below. Arrows indicate alterations identified in both STGD1 and AMD patients. Asterisks, missense mutations; D, deletions; S, splice donor site mutations; $\mathrm{X}$, stop codon-generating mutation. The number at the right signifies the last codon.

Table 2. Other variants in $A B C R$ (23).

\begin{tabular}{|c|c|c|c|}
\hline Alteration & AMD & STGD1 & $\begin{array}{c}\text { General } \\
\text { population }\end{array}$ \\
\hline V643G & $1 / 167 \quad(0.6 \%)$ & 0/98 (0\%) & $1 / 80 \quad(1.25 \%)$ \\
\hline $\mathrm{D} 846 \mathrm{H}$ & $0 / 167 \quad(0 \%)$ & $1 / 98 \quad(1 \%)$ & $1 / 50 \quad(2 \%)$ \\
\hline G863A & $1 / 167 \quad(0.6 \%)$ & $13 / 150(8.7 \%)$ & $2 / 220(0.9 \%)$ \\
\hline $\mathrm{R} 943 \mathrm{Q}$ & $6 / 127 \quad(4.7 \%)$ & $4 / 47(9.5 \%)$ & $13 / 80(16.25 \%)$ \\
\hline S2255I & $24 / 167(14.4 \%)$ & $8 / 98 \quad(8 \%)$ & $6 / 58(10.3 \%)$ \\
\hline
\end{tabular}


which map primarily to the highly conserved ATP-binding regions of the ABCR protein, AMD alterations were found outside these domains (Fig. 1). We have not found more than one AMD-associated variant in $A B C R$ in any $A M D$ proband, which is consistent with a dominant susceptibility locus. A thorough comparison of the genotypes and the phenotypes of large numbers of both STGD1 and AMD patients will be required to determine the significance of this observation and to clarify further the inheritance pattern of these alterations.

Most alterations detected in AMD patients were missense mutations outside of the ATP-binding domains. Conceivably, these variant proteins could accumulate in the rods and, in combination with age and other environmental factors, predispose carrier individuals to macular degeneration late in life. $A B C R$ mutations are associated with a wide range of clinical presentations. For example, patients with late-onset Stargardt disease were originally diagnosed with fundus flavimaculatus (25), a disease later defined to be allelic to STGD1 $(13,26)$. In addition, one form of autosomal recessive retinitis pigmentosa (RP19) maps to exactly the same chromosomal region as STGD1 and shares some phenotypic features with STGD1, which suggests that it might also be caused by an ABCR mutation (27).

The ABCR protein is identical to rim protein $(\mathrm{RmP})$, an abundant protein of un-

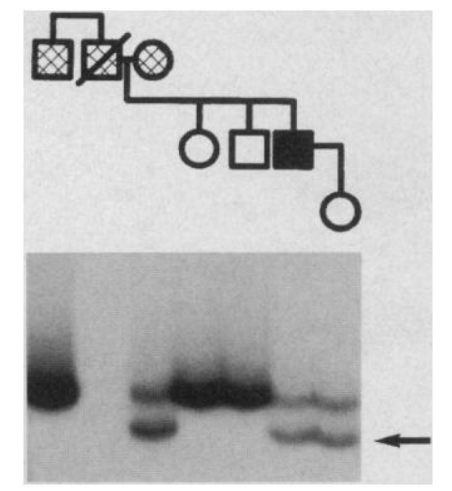

Fig. 2. Pedigree segregating $A M D$ and STGD1. An STGD1 patient (solid square) is shown, whose parents and paternal uncle all had AMD (hatched symbols). PCR products of $A B C R$ exon 49 from the double-stranded region of an SSCP/HA gel are shown below the pedigree. The arrow at right indicates the $6519 \Delta 11 \mathrm{bp}$ mutation in the individual with STGD1. He was diagnosed with STGD1 at age 38 , and the relatives with AMD were diagnosed between ages 55 and 70 . The mutation was inherited from his mother but was not found in either of his unaffected siblings (open symbols). His daughter also inherited the deletion, and at age 35 has normal vision and a normal retinal examination. A mutation in the proband's father has not yet been identified. known function that colocalizes with peripherin/RDS to the rod outer segment (16, $17,28)$. ABCR binds ATP, which is consistent with its role as an active transporter (29). ABCR is phosphorylated in response to light, which suggests that it plays a role in phototransduction (30). It has been hypothesized that $A B C R$ excretes metabolic products of phototransduction or lipids used by the rod outer segments $(12,16,17)$.

The discovery of specific ABCR alterations in AMD patients may permit presymptomatic screening of high-risk individuals, which in turn may facilitate earlier diagnosis and clinical testing of preventive and therapeutic strategies. Functional studies of this $\mathrm{ABC}$ transporter, including the identification of its substrate, may facilitate the development of drugs that modulate or prevent AMD. The identification of a large number of rare variants in this single gene in a common disease suggests that other complex human disorders may result from mutations in a gene responsible for a rare Mendelian condition with a similar phenotype.

\section{REFERENCES AND NOTES}

1. National Advisory Eye Council, Vision Research, a National Plan, 1994-1998 (NIH publication 93-3186 National Institutes of Health, Bethesda, MD, 1993).

2. R. Klein, B. E. K. Klein, K. L. P. Linton, Ophthalmology 99,933 (1992).

3. K. M. Egan and J. M. Seddon, in Principles and Practices of Ophthalmology, D. M. Albert and F. A Jakobiec, Eds. (Saunders, Philadelphia, 1993) chap. 109

4. N. M. Bressler, S. B. Bressler, S. L. Fine, Surv. Oph thalmol. 32, 375 (1988)

5. J. D. M. Gass, Arch. Ophthalmol. 90, 206 (1973).

6. J. M. Seddon, W. C. Willett, F. E. Speizer, S. E. Hankinson, J. Am. Med. Assoc. 276, 1141 (1996).

7. J. M. Seddon, U. A. Ajani, B. D. Mitchell, Am. J. Ophthalmol. 123, 199 (1997).

8. I. M. Heiba, R. C. Elston, B. E. K. Klein, R. Klein, Genet. Epidemiol. 11, 51 (1994).

9. B. H. F. Weber, G. Vogt, R. C. Pruett, H. Stohr, U. Felbor, Nature Genet. 8, 352 (1994)

10. M. A. De La Paz, M. A. Pericak-Vance, F. Lennon, J. L. Haines, J. M. Seddon, Invest. Ophthalmol. Vis. Sci. 38, 1060 (1997).

11. U. Felbor, D. Doepner, U. Schneider, E. Zrenner B. H. F. Weber, ibid., p. 1054.

12. R. Allikmets et al., Nature Genet. 15, 236 (1997).

13. K. L. Anderson et al., Am. J. Hum. Genet. 57, 1351 (1995).

14. R. Allikmets, B. Gerrard, A. Hutchinson, M. Dean Hum. Mol. Genet. 5, 1649 (1996).

15. D. S. Papermaster, C.A . Converse, M. Zorn, Exp. Eye Res. 23, 105 (1976).

16. M. Illing, L. L. Molday, R. S. Molday, J. Biol. Chem 272, 10303 (1997)

17. S. M. Azarian and G. H. Travis, FEBS Lett. 409, 247 (1997).

18. Age-Related Eye Disease Study, Manual of Procedures (National Eye Institute, Bethesda, MD, 1992).

19. Patients were selected from ophthalmic clinic populations (the Massachusetts Eye and Ear Infirmary Boston, MA; and the Moran Eye Center-University of Utah, Salt Lake City, UT). AMD was defined as ophthalmoscopically identifiable macular pathology, including macular drusen, RPE detachment, geographic atrophy, exudative AMD including RPE degraphic atrophy, exudative AMD including RPE de-
tachment, choroidal neovascularization, or disciform scar (18). Individuals were excluded if they had other causes of disciform or exudative maculopathy similar to AMD, such as ocular histoplasmosis syndrome myopic chorioretinal degeneration without or with lacquer cracks, pseudoxanthoma elasticum and other associations of angioid streaks, basal laminar drusen, or post-traumatic choroidal ruptures. Also drusen, or post-traumatic choroidal ruptures. Also
excluded were individuals with any other retinal and retinal vascular pathology (retinitis pigmentosa, vascular occlusive disease, or diabetic retinopathy). The retinal photographs and fluorescein angiograms of every patient with an $A B C R$ alteration were reviewed in a masked fashion with those of matched individuals without alterations to confirm assignment of affected status and to ensure exclusion of Stargardt disease. STGD1 patients were evaluated as in (13). Controls were U.S. Caucasians from the general population collected for other studies. Among the 167 patients, $59(36 \%)$ were male and $108(64 \%)$ were female; one was African-American and the remainder were European-American. Of the 26 individuals with AMD-associated alterations, $25 \%$ were male and $75 \%$ were female.

20. The exon-intron structure of the $A B C R$ gene was determined, primers were designed, and mutation screening and sequencing were performed as in (12). Primer sequences and polymerase chain reaction (PCR) conditions have been submitted to the Genome Data Base (http://gdbwww.gdb.org).

21. R. Allikmets et al., unpublished data.

22. Among all variants found in less than $1 \%$ of controls, there were 27 out of $167(16 \%)$ alterations in AMD patients and $3 / 220$ in controls $\left(P<10^{-7}\right)$. Considering only alterations also found in STGD1 patients (G863A, R1898H, G1961E, and 6519del11) (23) and D2177N yields $16 / 167$ in AMD patients and $3 / 220$ in controls. A total of $21 \mathrm{AMD}$ patients but only $1 \mathrm{con}$ trol had nonconservative $A B C R$ alterations (charge alterations, splice site variants, or frame shifts). The aggregate frequency of all variants was 35\% in AMD patients and $31 \%$ in controls. Whether the more common $A B C R$ variants affect function remains to be determined.

23. Single-letter abbreviations for the amino acid residues are as follows: A, Ala; D, Asp; E, Glu; F, Phe; G, Gly; H, His; I, Ile; K, Lys; L, Leu; M, Met; N, Asn; R, Arg; S, Ser; and T, Thr.

24. The frequency of STGD1 $(1$ in 10,000) yields a carrier frequency of $2 \%$. The assumption that approximately $20 \%$ of Caucasians develop AMD by age 75 and that $15 \%$ have $A B C R$ alterations yields a population frequency of $A M D$-associated alterations of $3 \%$. However, all STGD1 alleles may not predispose to AMD or vice versa. For example, the G863E alteration is commonly found in STGD1 patients but is not elevated in AMD patients versus controls (Table 2).

25. G. A. Fishman, Arch. Ophthalmol. 94, 2061 (1976).

26. S. Gerber et al., Am. J. Hum. Genet. 56, 396 (1995).

27. A. Martinez-Mir et al., Genomics 40, 142 (1997).

28. H. Sun and J. Nathans, Nature Genet. 75, 15 (1997)

29. T. A. Shuster, A. K. Nagy, D. B. Farber, Exp. Eye Res. 46, 647 (1988).

30. E. Z. Szuts, Biochemistry 24, 4176 (1985).

31. We thank the participating patients, their families, and their physicians for cooperation; A. Crandall, M. Swartz, and M. Teske at the Moran Eye Center; M. Sullivan and C. Tucker at the Massachusetts Eye and Ear Infirmary; S. Cevario and R. Workman for technical assistance; G. Huttley for statistical advice; and the University of Utah Health Sciences Sequencing Facility. Supported by grants from the Foundation Fighting Blindness (R.A.L. and J.R.L.), Research to Prevent Blindness (R.A.L., J.M.S., P.S.B., and N.A.Z.), the Steinbach Fund (J.R.L. and R.A.L.), the W. M. Keck Foundation (M.L. and A.P.), the Macular Degeneration Foundation of Boston (J.M.S.), the Tornquist Charitable Foundation (M.L.), and the National Eye Institute (J.R.L., R.A.L., J.M.S., and N.F.S.). The content of this publication does not necessarily reflect the views or policies of the Department of Health and Human Services, nor does mention of trade names, commercial products, or organizations imply endorsement by the U.S. government.

19 June 1997; accepted 11 August 1997 